\title{
Extra-Cardiac Fetal Rhabdomyoma of Dorsum of Foot: A Rare Case Report
}

\author{
Junu Devi ands Deepjyoti Kalita \\ Department of Pathology, Gauhati Medical College, Guwahati. Assam. India
}

\begin{abstract}
Extracardiac fetal rhabdomyoma is a rare tumor occurs almost exclusively in children under the age of 10 years with a predilection for the head and neck region and chest wall. It can occur in other sites also. However it is very rare in dorsum of foot. Rhabdomyoma accounts for less than $2 \%$ of all striated muscle tumors. Differentiation from Embryonal Rhabdomyosarcoma(ERMS) is very important to avoid aggressive treatment and its consequences. We have reported this case of extracardiac fetal rhabdomyoma of dorsum of foot in an eleven month old male child because of rarity of location which was surgically resected. Histopathology and immunohistochemistry confirmed the diagnosis of extra cardiac fetal rhabdomyoma.
\end{abstract}

Keywords: Rhabdomyoma, Extracardiac, Foot, Histopathology, Immunohistochemistry.

\section{Introduction}

Extracardiac fetal rhabdomyoma are extremely rare benign tumor of striated muscle comprising of less than $2 \%$ of all striated muscle tumors. ${ }^{[1]}$ It affects both children and adult that are composed of immature striated muscle at the $6^{\text {th }}$ to $10^{\text {th }}$ week stage of development. ${ }^{[2]}$ It usually occurs in the head and neck region, however, it has been identified in wide array of anatomic sites. ${ }^{[2]}$ These tumors are adequately treated with complete excision, local recurrence possible if incompletely removed. Some fetal rhabdomyoma may reccur as rhabdomyosarcoma. ${ }^{[1]}$ Extracardiac fetal rhabdomyoma of dorsum of foot is very rare. We report this case of fetal rhabdomyoma arising in the dorsum of foot with complete resection in a 11 month old boy which was clinically and radiologically suspected as rhabdomyosarcoma .

\section{Case Report}

An eleven month old boy was referred to the department of Paediatric surgery at Gauhati Medical College and Hospital (GMCH) for a small subcutaneous mass at the dorsum of right foot which was initially noticed by the mother and later on she informed paediatrician during child's regular checkup. On examination a 4.5 to $4.0 \mathrm{~cm}$ swelling was noted on dorsum of right foot which was painless. Child was admitted in the paediatric surgery department for evaluation. All laboratory tests were within normal limit. HR Ultrasonography and plain x-ray identified a small nodular mass located superficially and a provisional diagnosis of rhabdomyosarcoma was made.

The child underwent complete local excision of the mass and it was sent to the pathology department GMCH for histopathological examination.Grossly it was well circumscribed $(4.0 \times 3.5 \mathrm{~cm})$ (Fig.1) whitish mass with myxoid cut surfaces. Histopathological findings were of a well circumscribed cellular lesion composed of oval to spindle shaped cells with moderate amount of cytoplasm and bland nuclei in a variable fibromyxoid stroma (Fig.2a,b,c). Some ganglion like rhabdomyoblasts was also noted. Few cells showed cytoplasmic cross striations suggestive of rhabdomyoblastic differentiation. Mitotic figures were absent and neither cellular atypia nor immature looking cells were noted. Immunohistochemistry showed positivity for desmin (Fig3). Histopathological and immunohistochemical findings were consistent with a myxoid form of fetal rhabdomyoma. So diagnosis of extracardiac fetal rhabdomyoma was made. Post operative period was uneventful and patient was discharged and regular followup was adviced. Till date patient is doing well without any evidence recurrence.

\section{Discussion}

Rhabdomyomas (RM) are benign neoplasms found more frequently in the myocardium than in the striated muscles. There are two main types, cardiac and extracardiac, with extracardiac consisting of three clinical and histologic subtypes: adult, fetal and genital accounting for 50, 40, and $10 \%$ of tumors respectively. ${ }^{[3]}$ Fetal $\mathrm{RM}$ are benign tumor with skeletal muscle differentiation mostly occur in head and neck region. It is observed in higher incidence in male children these includes newborn infant or very young children. There is no known ethnic or racial preference. No definite risk factors have been identified,however it is thought to be associated with nevoid basal cell carcinoma syndrome. Currently exact cause and mechanism of 


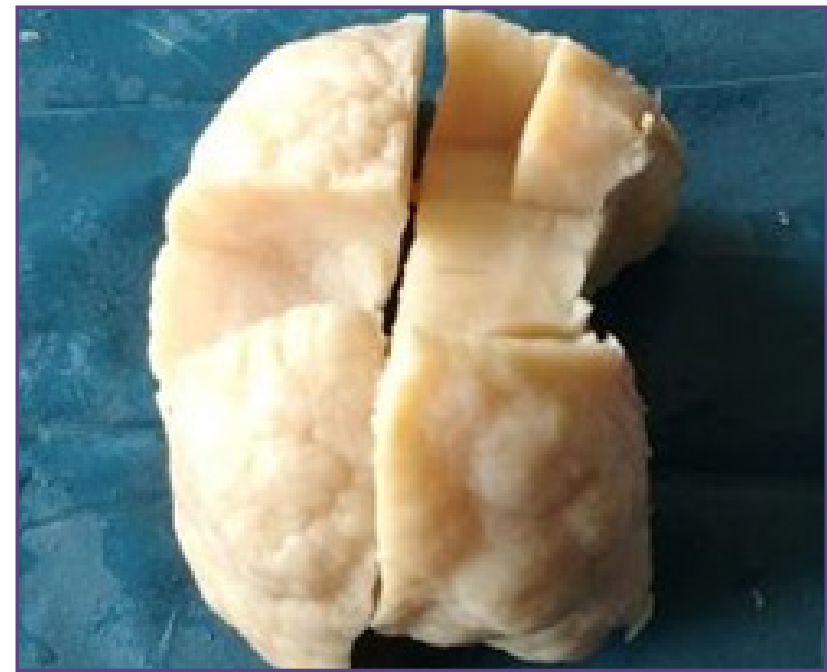

Fig.1: Gross photograph of Rhabdomyoma.

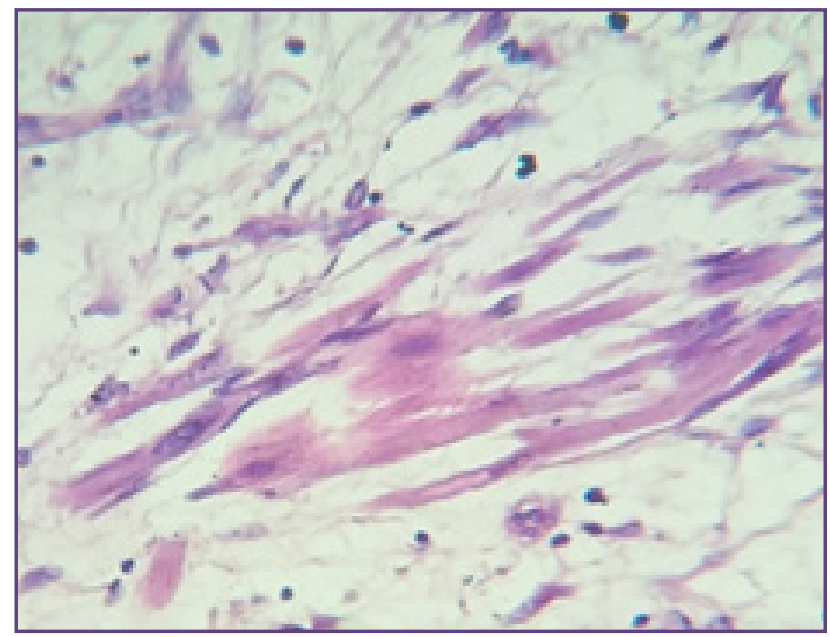

Fig. 2(b): Photomicrograph showing cross striations in differentiating muscle fibers (H\&E,10x40).

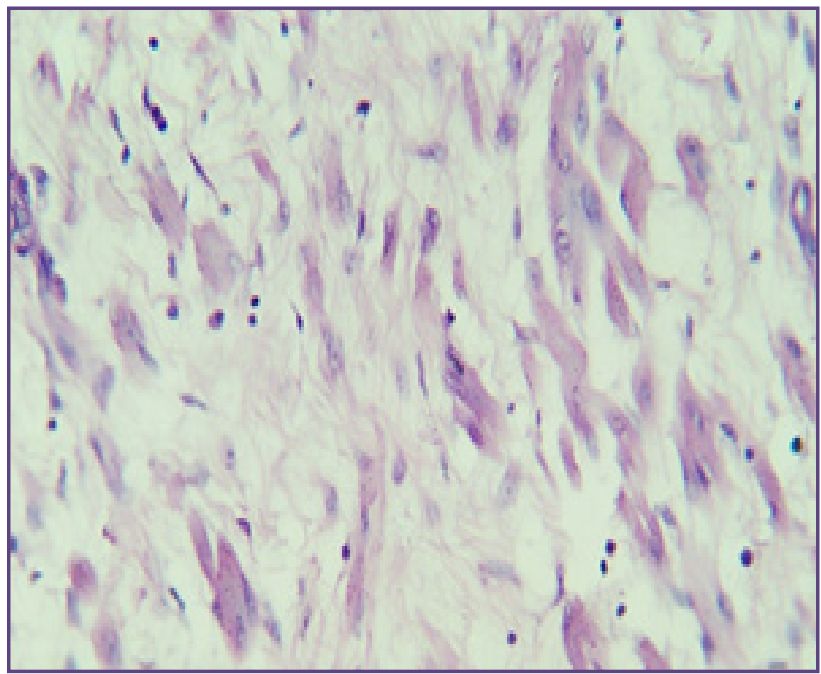

Fig. 2 (a): Photomicrograph showing immature skeletal muscle fibers .(H\&E, 10x40).

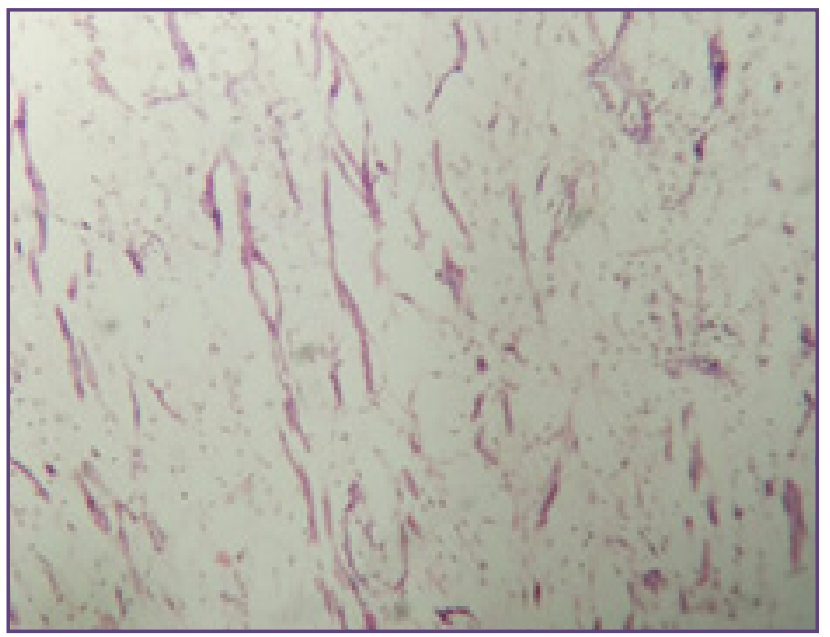

Fig. 2(c): Photomicrograph showing developing myotubes (H\&E,10x10).

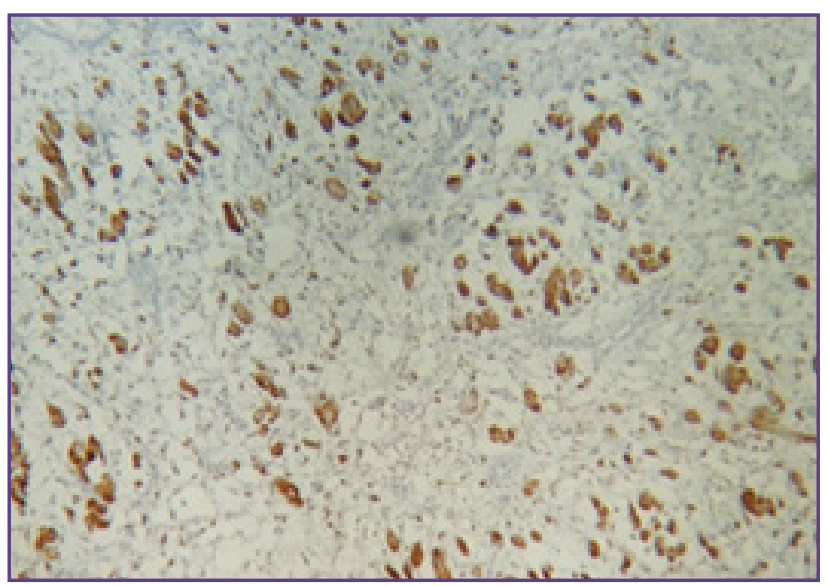

Fig. 3: Photomicrograph showing Desmin positivity (IHC, 10X10). 
formation of fetal RM is unknown. It is thought to occur due to certain genetic aberration or some morphological abnormalities in the tissue. Signs and symptoms are related to sites, when occur in the oral cavity and upper respiratory tract may cause breathing difficulties. ${ }^{[4]}$ In children approximately two dozen cases of cervicofacial extracardiac fetal RM have been reported in the literature to date and a few cases have been identified in other anatomic sites, such as lower extremity, ${ }^{[5]}$ the abdominal wall ${ }^{[6]}$ or retroperitoneum ${ }^{[7]}$ and mediastinum. ${ }^{[8]}$ We report this rare case because of unusual location.

Histologically fetal RM composed of elongated spindle shaped skeletal muscle element in varying stage of differentiation. There are two subtypes of fetal RM, "classic" with immature histology and "intermediate" with more rhabomyoblastic differentiation. However several tumors show overlapping features. ${ }^{[0]}$ The differential diagnosis includes rhabdomyosarcoma (RMS) and benign hamartomatous lesions, such as neuromuscular hamartomas of the skin. ${ }^{[10,11]}$ Distinction from spindle cell variant of embryonal RMS and other RMS may be difficult.Therefore it is very important to make an accurate diagnosis for proper treatment particularly when it occurs in sites other than head and neck region. Rhabdomyosarcomas lack circumscription have infiltrative margins, and invade normal tissue, show less cellular differentiation and manifest mitotic figures or necrosis. ${ }^{[12]}$ Fetal RM have variable cellularity with differing degrees of striated muscle differentiation and have a range of cell types. ${ }^{[3]}$ They rarely show areas of necrosis, nuclear atypia and lack hypercellularity, abnormsally distributed chromatin and absent or low mitotic figure. ${ }^{[12]}$ Desmin is the most reliable immunohistochemical marker for cells with skeletal or smooth muscle differentiation. Other marker like vimentin, myoglobin, muscle specific actin also give positive reaction. ${ }^{[13]}$ Other differential diagnosis are granular cell tumor, hibernoma, paraganglioma, PEcoma, histiocytic tumor. Presence of myogenic markers and standard clinicopathological evaluation is sufficient for their unequivocal separation. ${ }^{[14]}$

Complete surgical removal is mandatory and follow up care with regular screenings are important because fetal RM may recur and sometime give rise to rhabdomyosarcoma. Prognosis of fetal RM is excellent with complete surgical removal. Incomplete removal increases the chance of recurrence.No instance of aggressive local tumor growth or metastasis has been documented. Local tumor recurrence has been reported usually attributed to incomplete resection. ${ }^{[15]}$
Possibility of fetal rhabdomyoma should be considered in differential diagnosis of superficial swelling in foot in neonate

\section{Conclusion}

Extracardiac fetal rhabdomyoma is a rare tumor, complete surgical removal reduces the risk of recurrence. Accurate diagnosis and distinction from embryonal RMS is very much essential for unwanted aggressive treatment. Histopathology and immunohistochemistry gives confirmatory diagnosis.

\section{Reference}

1. Gattuso P, Reddy BV, David O,Spitz DJ,Haber MH .Differential Diagnosis in Surgical Pathology 3rd edition, Philadelphia, Elsevers saunders,2015.

2. Walsh SN et al. Cuteneousfetal Rhabdomyoma: A case report and Historical review of the literature. The American Journal of Surgical Pathology, March 2008;32: 485-491.

3. Zolota V, Tzelepi V, Charoulis N, Apostolakis E, Dougenis D. Mediastinal rhabdomyoma: case report and review of the literature. Virchows Arch 2006:449:124-8

4. Gajda MC. Halzhausen HJ, Jamali Y, Schrom T, Hauptmann. S, Bloching M. Multifocal adult Rhabdomyoma of the neck ; a rare entity]. Otolaryngol 2005;59:883-886.

5. Premalata CS, Kumar RV, Saleem KM, Fatima IJ, Das K. Fetal rhabdomyoma of the lower extremity. Pediatr blood Cancer 2009;52:881-3

6. KonradEA, MeisterP,HubnerG.Extracardiacrhabdomyoma: report of different types with light microscopic and ultrastructural studies. Cancer 1982:49:898-907.

7. DiSanto S. Abt AB, Boal DK, Krummel TM. Fetal rhabdomyoma and nevoid basal cell carcinoma syndrome. PediatrPathol 1992: 12: 441-7

8. Go Miyano,Hiromu Miyake,Naoto Urushihara et al . Mediastinal extracardiac fetal rhabdomyoma;a case report. Journal of paediatric surgery Case reports, April 2014;2(4):196-199 .

9. Kapadia SB, Meis JM, Frisman DM, Elles GL, Heffner DK. Fetal rhabdomyomas of the head and neck: A clinicopathologic and immunophenotypic study of 24 cases. Hum Pathol 1993; 24: 754-765.

10. Cavazzana AO, Schmidt D, Ninfo V, Marms D, Tallot M, Carli M, et al . Spindle cell rhabdomyosarcoma. Am J SurgPathol 1992; 16:229-235.

11. Sahn EF, Garen PD, Pai GS, Levkoff AH, Hagetty RC, Maize JC. Multiple rhabdomyomatous mesenchymal hamartoma of skin Am J Dermatopathol 1990; 12:485-491.

12. Dehner LP, Crotty PL, Nakhieh RF. Juvenile rhabdomyomas . More than a fetal but less than adult rhabdomyomas. Lab Invest $1992 ; 5: 4 \mathrm{P}$. 
13. Hanper K, RenninghoftJ,Schaffer H. Rhabdomyoma of the larynx recurring after 12 years: immunohistochemistry and differential diagnosis. Arch Otorhinolaryngol 1989; 246: 222-226

14. David J. Dabbs. Diagnostic Immunohistochemistry,
Theranostic and Genomic Application 4th Edition, Philadelphia, Elsevier saunders ,2014.

15. T.AValdez,U.Desai M.S volk. Reccurentfetal Rhabdomyoma of head and neck. Int. J pediatricOtorhinolaryngol, 2006;70:1115-8.

*Corresponding author:

Dr Junu Devi.C-3 Kanika Apartment,Noonmati,Lastgate, Guwahati -20. Assam. India.

Phone: +919435144568.

Email: drjdevipath@gmail.com

Date of Submission : 23.08.2016

Date of Acceptance : 27.12.2016

Financial or other Competing Interests: None.

Date of Publication : 23.02.2017

Annals of Pathology and Laboratory Medicine, Vol. 04, No. 01, January - February, 2017 